\title{
Phacoemulsification in Angle Closure Glaucoma
}

\section{Jovina See}

Glaucoma Service, National University Hospital, Singapore

\section{INTRODUCTION}

Phacoemulsification in angle closure can be challenging. Many factors, including large lens, small pupil and a narrow working space in the anterior chamber, contribute towards an increased level of difficulty. Angle closure eyes are also often associated with increased risk of complications such as malignant glaucoma and suprachoroidal hemorrhage.

For the inexperienced or the unsuspecting, phacoemulsification in these eyes can often result in unnecessary and devastating complications, including posterior capsule rupture, dropped nucleus, or suprachoroidal hemorrhage. This chapter therefore aims to identify the surgical challenges presented in eyes with angle closure, and to suggest interventions and surgical techniques that may be useful in ensuring a smooth and uncomplicated surgical course during phacoemulsification.

Before we come to this, it is first necessary to look at the aims of and indications for cataract extraction in angle closure.

\section{Aims of Phacoemulsification in Angle Closure}

1. Improve visual acuity (by removal of the cataract).

2. Widen the anterior chamber angle to decrease the risk of angle closure.

3. Break an attack of acute angle closure that is medically unresponsive or not amenable to laser peripheral iridotomy.

4. Possibly reduce the likelihood of further peripheral anterior synechiae formation and progression onto chronic angle closure glaucoma.

\section{Indications for Phacoemulsification in Angle Closure (Flow Chart 1)}

\section{Absolute indications:}

1. Secondary angle closure:

a. Phacomorphic glaucoma (Fig. 1)

b. Subluxated lens

c. Neovascular glaucoma, where cataract is so dense as to preclude laser pan-retinal photocoagulation or monitoring of diabetic retinopathy.
2. Acute primary angle closure that is unresponsive to medical and laser treatment, including laser iridoplasty and iridotomy (Fig. 2).

\section{Relative indications:}

3. Cataract that is visually significant in an eye with angle closure.

4. Primary angle closure suspect or primary angle closure where lens is thick or anteriorly positioned so, that the angle remains occludable despite adequate laser iridotomy and laser iridoplasty.

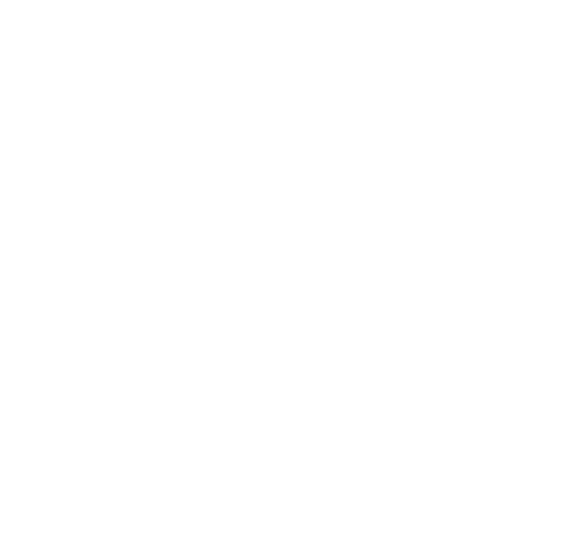

Fig. 1: Phacomorphic glaucoma

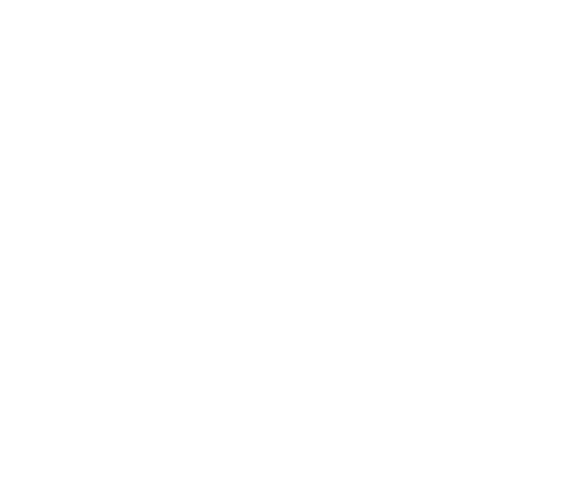

Fig. 2: Acute angle closure attack 


\section{Preoperative Considerations}

1. When should you proceed with surgery? (See algorithm below).

2. Laser iridotomy (LI) $[ \pm$ laser iridoplasty (LIP) in cases of angle crowding due to peripheral iris roll or plateau iris] should be performed in all cases of angle closure and angle closure glaucoma (unless surgery for visually significant cataract is imminent) (Figs 3 and 4).

3. Careful evaluation and identification of risk factors. For example, state of corneal health, evidence of lens subluxation (e.g. Asymmetric anterior chamber depth, iridodonesis, phacodonesis, lens tilt. Ultrasound biomicroscopy (UBM) is useful in such cases (see algorithm below)), previous occurrence of malignant glaucoma or suprachoroidal hemorrhage in the fellow eye.

4. Prudent counseling regarding possible intraoperative and postoperative complications, as well as visual prognosis.

5. Combined phacoemulsification with intraocular lens implant and glaucoma filtration surgery should be considered where intraocular pressures are inadequately controlled despite maximal medical treatment, or where there is evidence of progressive glaucomatous optic neuropathy, especially in cases where noncompliance is suspected.

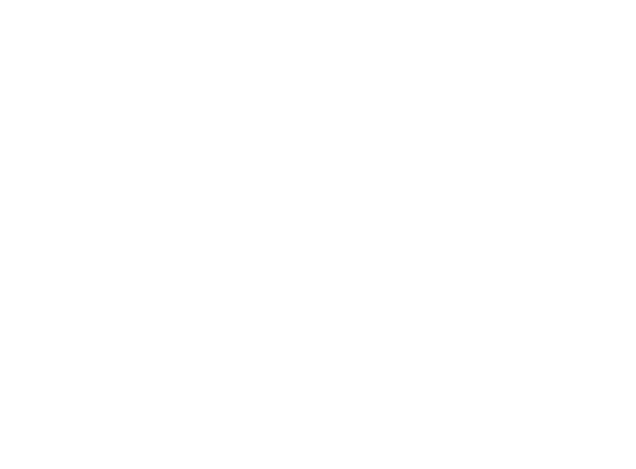

Fig. 3: Laser peripheral iridotomy

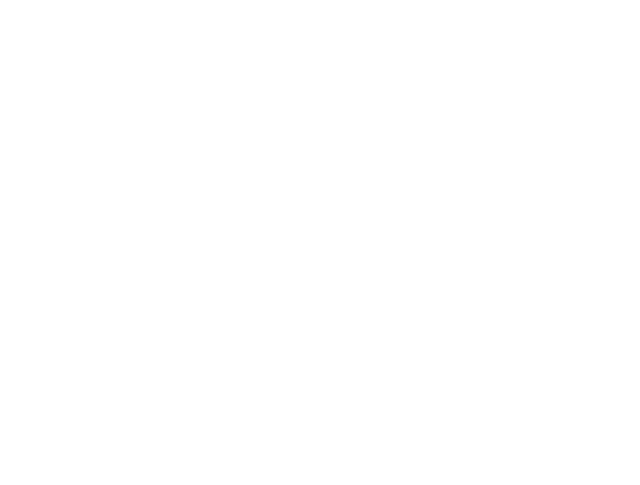

Fig. 4: Laser iridoplasty

Flow Chart 1: Management of angle closure

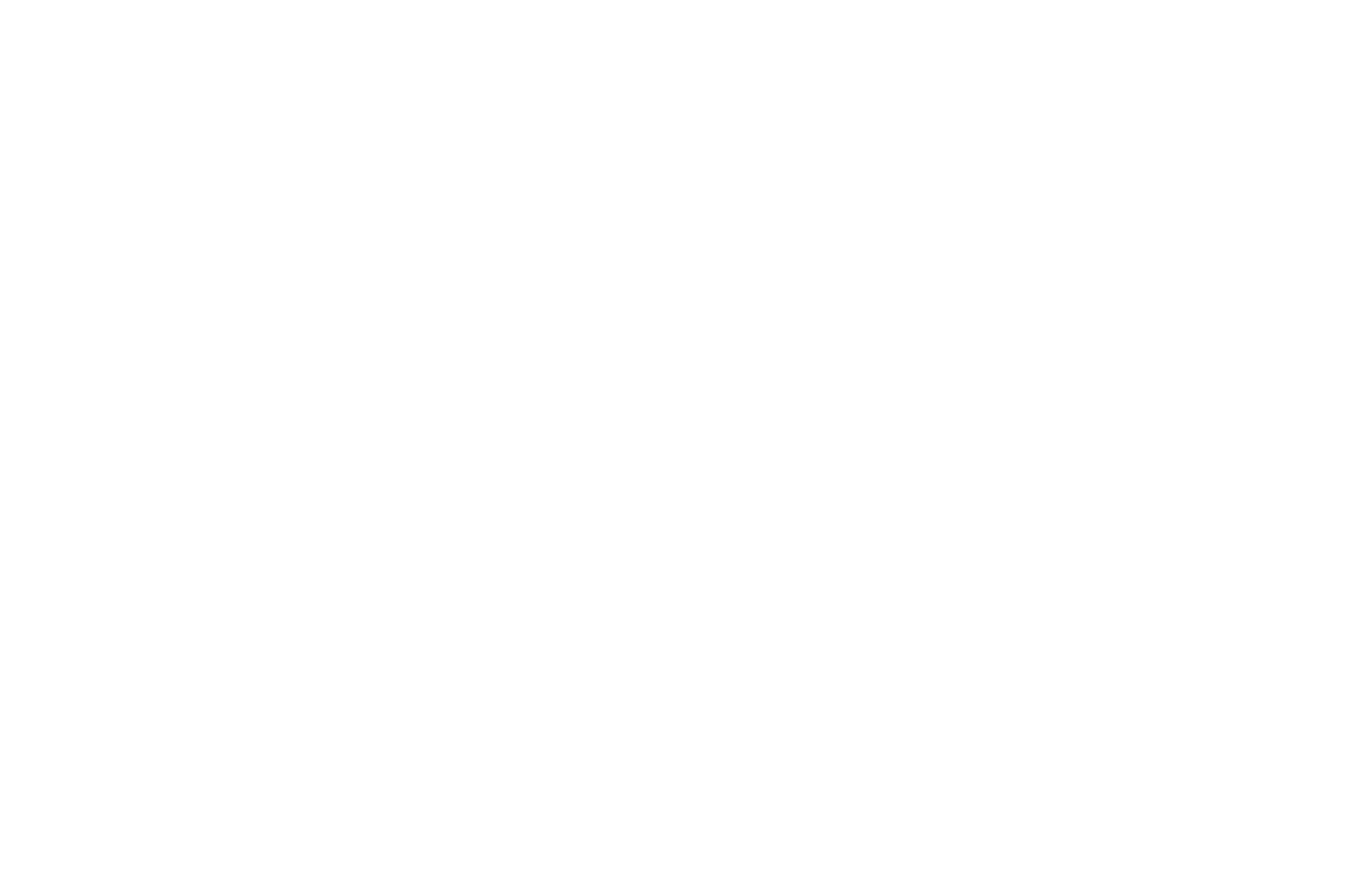




\section{Surgical Challenges}

Phacoemulsification in angle closure eyes, whether in the acute or nonacute setting, presents many surgical challenges. Recognizing these challenges will help in the planning of surgical technique, and hence reduce the risk of intraoperative complications. These challenges are listed below:

1. Small palpebral aperture - difficult access.

2. Inflamed eye - especially in eyes with uncontrolled raised intraocular pressures, or eyes on chronic medical treatment (Fig. 5).

3. High IOP - in acute angle closure and chronic angle closure glaucoma.

4. Poor visibility, diminished red reflex - if corneal edema is present due to high pressures.

5. Corneal epithelial and stromal edema - propensity to Descemet's tear, corneal decompensation.

6. Reduced working space due to a shallow anterior chamber (Fig. 6).

7. Small pupil - if atrophy of dilator pupillae due to previous attack of acute angle closure, or if on prolonged miotic treatment. A small pupil may also be the result of posterior synechiae, which may be diffusely present over a large area beyond the pupil margin.

8. Flaccid iris, iris prolapse.

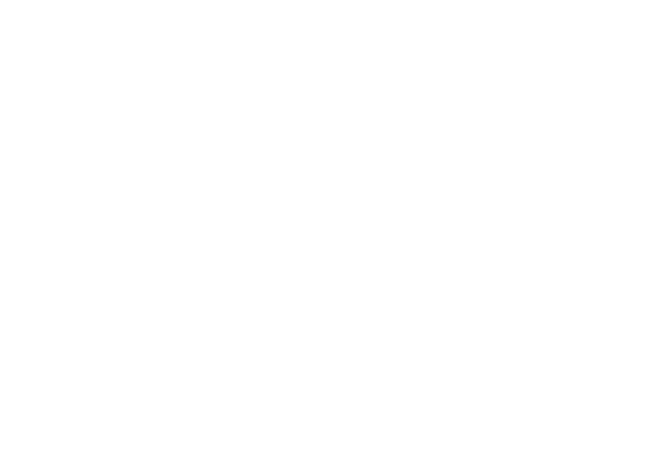

Fig. 5: Inflamed eye in acute angle closure

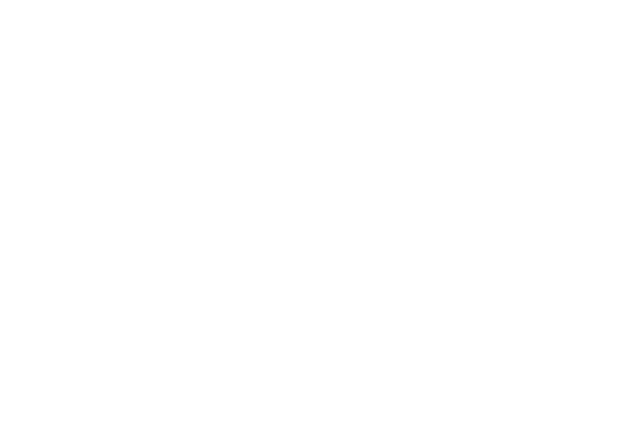

Fig. 6: Shallow anterior chamber
9. Peripheral anterior synechiae at the site of the corneal wound may interfere with entry into the anterior chamber.

10. Difficult capsulorrhexis.

11. Large, and often hard, lens.

12. High capsular bag tension.

13. Lens subluxation.

14. Increased risk of posterior capsule rupture and vitreous loss.

15. Increased risk of malignant glaucoma and suprachoroidal hemorrhage intraoperatively or postoperatively.

\section{Surgical Technique of Phacoemulsification in Angle Closure}

Knowing the potential difficulties and dangers we may face in the phacoemulsification of a cataract in an eye with angle closure, we can then modify our surgical technique to minimize the risk of intraoperative complications. The lens in angle closure is often large. Furthermore, coupled with the reduced working space, and a cornea that may already have been compromized by edema, phacoemulsification in angle closure necessitates a more patient and gentle approach, taking special care to respect the corneal endothelium during the procedure. Unnecessary entry into the eye should be avoided during the entire procedure, to minimize iatrogenic damage to an already unhealthy cornea that is prone to Descemet's tears and corneal endothelial decompensation.

\section{Some Useful Surgical Tips}

1. Control intraocular pressure as much as possible preoperatively.

- In an acute angle closure attack, the patient should have been given maximal medical treatment, including topical beta-blockers, prostaglandins, alpha-agonists, systemic acetazolamide (unless contraindicated) and topical anti-inflammatory steroids. A laser iridotomy, with or without the prior assistance of laser iridoplasty, should have been attempted and sufficient time given to allow resolution of the acute attack, prior to making the decision to proceed with surgery in such an inflamed eye. Only rarely attempts lens extraction under such urgent circumstances in order to break the attack. Operating on such high-pressured, inflamed eyes is associated with a higher risk of intraoperative suprachoroidal hemorrhage.

2. Pupil dilatation.

- Preoperatively, a combination of topical tropicamide $\mathrm{HCl}$ 1\% (q5 min × 3), phenylephrine 2.5\% (q5 min × 2), flurbiprofen $\mathrm{Na}$ 0.03\% (q10 min $\times 6$ ) and atropine sulphate $1 \%$ should be administered to allow maximal 
pupil dilation for ease of performing a capsulorrhexis and subsequent phacoemulsification. The pupil should be dilated just prior to commencement of surgery, in order to avoid the risk of establishing an acute angle closure attack.

3. Intravenous mannitol.

- Prior to commencement of surgery, consider giving $20 \%$ mannitol intravenously to reduce vitreous volume, so as to lower intraocular pressure and increase working space in the anterior chamber. The entire volume of mannitol should be administered before the start of surgery.

4. Consider trans pars plana aspiration of fluid from the vitreous.

- In an acute angle closure attack, fluid aspiration from the vitreous using a 23-G needle attached to a $2 \mathrm{ml}$ syringe passed via pars plana may be considered in order to attempt to deepen the anterior chamber, break the attack, and further reduce intraocular pressure, before entering the anterior chamber. This not only helps to increase the working space in the anterior chamber, and improve visibility by reducing corneal edema, it reduces the occurrence of iris prolapse during phacoemulsification.

5. Gradual decompression of the anterior chamber.

- An anterior chamber paracentesis wound is first made on the cornea and repeated controlled manual expression of aqueous from the anterior chamber is performed via this small opening. Ample time should be allowed for this procedure to allow gradual decompression of the anterior chamber, as sudden decompression is associated with a high-risk of suprachoroidal hemorrhage. Any pre-existing corneal edema is often observed to resolve at this point and visibility improved, as the intraocular pressure is lowered. Performing decompression through such a small wound also prevents iris prolapse, which may otherwise cause the iris to become flaccid and interfere with phacoemulsification later.

6. Injection of a very small volume of balanced saline solution with adrenaline 1:1000 via the anterior chamber paracentesis wound aids in pupil dilatation.

7. Proper wound construction.

- The main wound should be constructed carefully with a biplane incision. It should not be placed too posteriorly, as this will increase the likelihood of iris prolapse in an already atonic iris. It should provide a snug fit for the introduction of the phaco tip. This will minimize wound leak which may otherwise result in fluctuation of the anterior chamber.
8. Viscoelastic substances.

- A high-molecular-weight viscoelastic such as Healon 5 is next injected into the anterior chamber via the main wound to increase the working space in the anterior chamber as well to coat and help protect the corneal endothelium. Such a viscoelastic will also aid in further pupil dilatation. Viscoelastics may also be used to perform posterior synechialysis where necessary. However, thorough removal of such highviscosity viscoelastics at the end of surgery is necessary to avoid postoperative intraocular pressure spikes that may be detrimental to the final visual acuity achieved.

9. Pupil dilatation.

- In cases where posterior synechiae is present, viscoelastic substances are helpful in breaking the synechiae, and enlarging the pupil.

- With small pupils, additional mechanical pupil stretch in one or more meridian with a pair of Kuglen hooks may be necessary to facilitate capsulorrhexis and phacoemulsification.

- If pupil dilation is not maintained despite mechanical stretch, 4 iris hooks placed via 4 limbal paracentesis incisions may be useful. These hooks should be placed symmetrically so, that the pupil adopts a diamond configuration with a corner near the main wound to allow easy aspiration of subincisional cortex. The hooks should also be placed parallel to the iris plane to avoid anterior pull on the iris diaphragm.

- Pupil dilators (e.g. Beehler) and pupil ring expanders (e.g. Morcher, Becton-Dickinson) are less useful in angle closure eyes, as the reduced amount of working space often implies a higher risk of corneal endothelial damage during the insertion of these devices.

10. Decompress lens.

- Where the lens is intumescent, the cataract may first be decompressed by aspirating liquefied lens cortex with a 23-G needle that is used to penetrate the anterior lens capsule centrally, before capsulorrhexis is performed. This will increase the working space in the anterior chamber for phacoemulsification to proceed. It will also decrease the capsular bag tension, thereby reducing the risk of posterior capsule rupture and vitreous loss.

11. Capsulorhexis.

- The capsulorhexis should be sufficiently large to allow easy removal of segments of the often bulky lens. This reduces the amount of stress that would otherwise be placed on the capsular bag during phacoemulsification. 
- Be vigilant during capsulorrhexis for any phacodonesis that may suggest lens subluxation and the possible need for insertion of a capsular tension ring or even a posterior scleral-fixated intraocular lens with anterior vitrectomy.

12. Phacoemulsification.

- Bimanual phacoemulsification is a good technique to use in eyes with angle closure. The smaller wounds used in this technique reduces the likelihood of iris prolapse and allows greater stability of the anterior chamber. However, a hard cataract can be difficult to tackle with the bimanual irrigating chopper. In addition, the small-diameter phaco probe may be easily occluded by hard nucleus fragments. Wound gape may also result from excessive manipulation with the instruments.

- Angled phaco tips like the Kelman tip may be more difficult to use, especially in the initial stage of sculpting, due to the limited working space in the anterior chamber. A straighter phaco tip may be a better option.

- Particularly with a small pupil or capsulorrhexis, the initial sculpting should stop short of the iris and capsulorrhexis edge near the pupil plane. Only upon
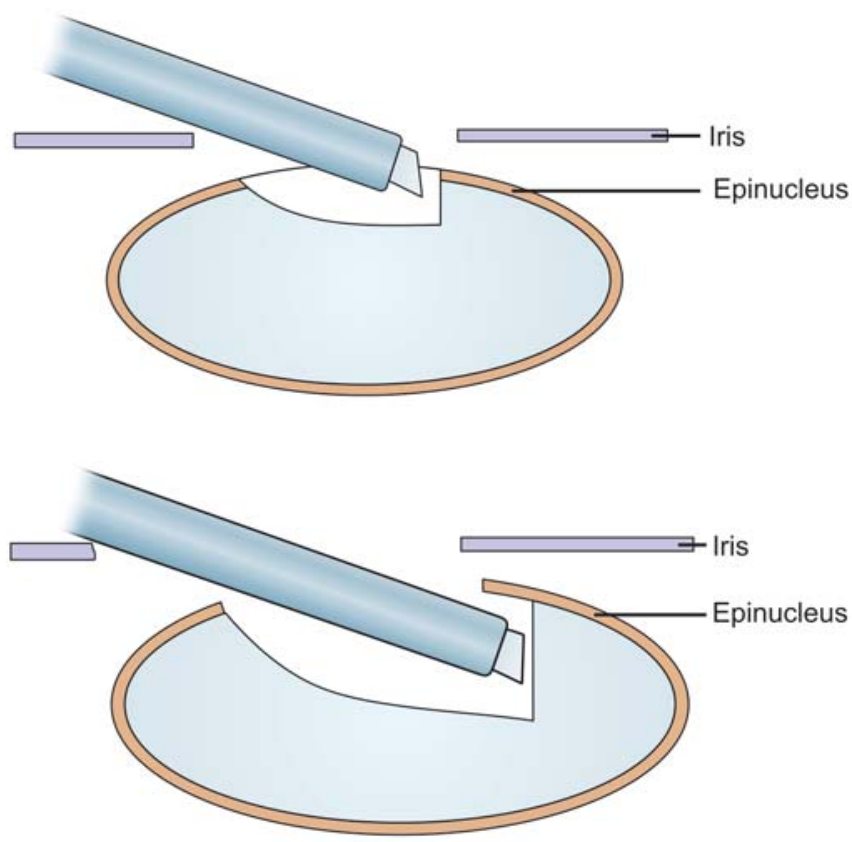

Fig. 7: Only upon advancing deeper, under the protective layer of anterior epinucleus, should sculpting progress more peripherally advancing deeper, under the protective layer of anterior epinucleus, should sculpting progress more peripherally (Fig. 7). This avoids trauma to the iris, which may otherwise cause the iris to become even more flaccid.

- Phacoemulsification of the lens should be performed in the capsular bag instead of in the shallow anterior chamber, to avoid mechanical and thermal damage to the corneal endothelium caused by the phacotip or lens fragments. A quick-chop technique where the lens nucleus is segmented at the center of the pupil is preferred, especially where the pupil or capsulorrhexis is small. As the lens is often large, patience should be exercised to fragment the lens into smaller segments for removal. All movements should be gentle to avoid unnecessary stress on the capsular bag and zonules. Care should be taken to minimise postocclusion surge. For soft cataracts, the hemi-flip technique can be very useful.

- As phacoemulsification progresses and more lens material is aspirated, the anterior chamber will deepen progressively, and the surgery should become easier. Relatively "cool” phaco tips such as Whitestar (Sovereign, with CASE and ICE technology) help to minimize thermal damage to the cornea. Care should also be taken to regularly wet the cornea, which will help to cool the phaco tip and further minimize thermal damage.

- After removal of a large lens, the stretched capsule is usually quite floppy at this stage. Caution should be exercised when performing irrigation and aspiration, as any surge can increase the risk of posterior capsule rupture.

13. Goniosynechialysis.

- Goniosynechialysis combined with phacoemulsification, with or without laser iridoplasty has been reported to be an effective treatment for synechial chronic angle closure glaucoma and cataract. ${ }^{1,2}$

14. Surgical peripheral iridectomy.

- This should be considered at the end of surgery in cases of acute angle closure where laser peripheral iridotomy was previously not possible, as these inflamed eyes tend to induce a significant amount of fibrin formation postoperatively which can sometimes lead to pupil block between the iris and intraocular lens.

15. Thorough removal of viscoelastics, especially if highviscosity substances used.

- Retention of high-viscosity viscoelastic substances, e.g. Healon 5 can result in postoperative intraocular 
pressure spikes which if unrecognized, can lead to irreversible glaucomatous optic neuropathy. Therefore, intraocular pressures must be measured postoperatively, and the responsible viscoelastics removed either by posterior lip pressure via the anterior chamber paracentesis wound, or even returning to the operating theatre to perform a simple Simcoe removal.

16. Postoperative care.

- Frequent dosing of topical steroids, especially in eyes operated during the acute attack of angle closure

- Careful monitoring of postoperative intraocular pressure, which may be raised due to retained viscoelastics, increased inflammation or released iris pigment

- Be vigilant for the occurrence of malignant glaucoma, which has been reported to occur in 0.6 to $4 \%$ of cases of angle closure glaucoma. ${ }^{3,4}$ Malignant glaucoma typically presents with high intraocular pressure accompanied by shallowing of central and peripheral anterior chamber, in the presence of a patent iridotomy or iridectomy.

\section{Possible Complications Associated with Phacoemulsification in Angle Closure}

1. Corneal endothelial damage, Descemet's tears and corneal decompensation (Fig. 8).

2. Posterior capsule rupture.

3. Dropped nucleus.

4. Vitreous loss.

5. Suprachoroidal hemorrhage.

6. Intraoperative or postoperative intraocular pressure spikes.

7. Malignant glaucoma (Fig. 9).

8. Cystoid macular edema - The glaucoma patient is at a higher risk of postoperative cystoid macular edema because

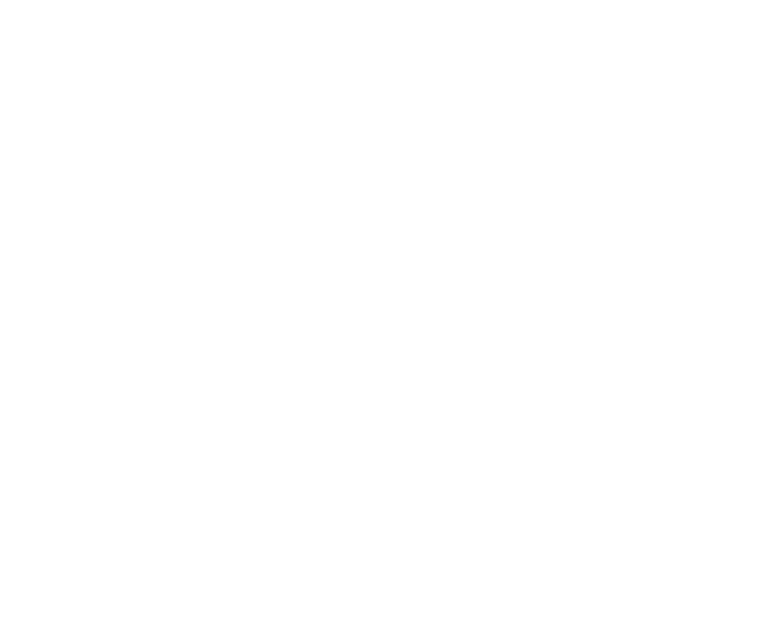

Fig. 8: Corneal decompensation
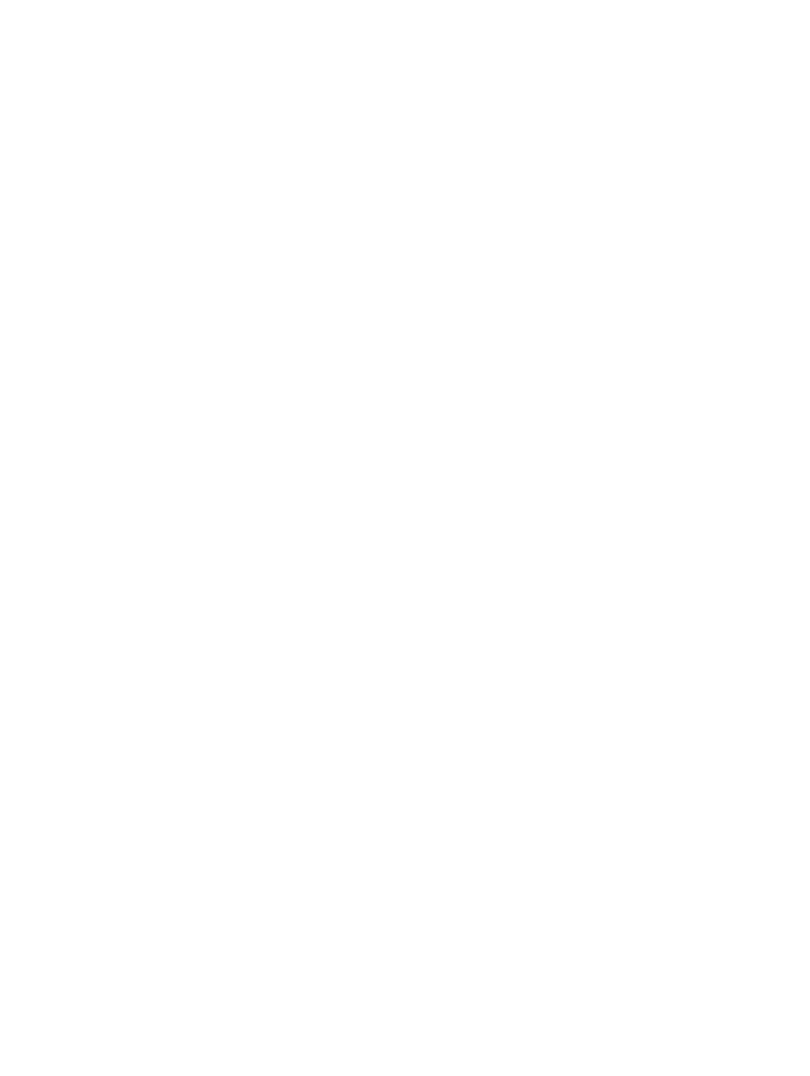

Fig. 9: Malignant glaucoma imaged by UBM: Central image showing central anterior chamber shallowing, peripheral image showing closed anterior chamber angle, and slight supraciliary effusion

of their tendency toward greater postoperative inflammation, as well as their use of medications like latanoprost, which should be discontinued. Topical nonsteroidal anti-inflammatory medications may be helpful in such cases.

9. Endophthalmitis.

10. Postoperative progressive chronic angle closure glaucoma.

\section{CONCLUSION}

Phacoemulsification in angle closure offers a chance of visual rehabilitation in patients with significant cataracts. It also enables a decrease in intraocular pressures to normal levels if performed early in a patient with primary angle closure. If performed before the formation of a significant amount of peripheral anterior synechiae, phacoemulsification results in significant increases in both depth of the anterior chamber as well as width of the anterior chamber angle ${ }^{5}$ (Figs 10A to C). This decreases the likelihood of further formation of peripheral anterior synechiae, and hence progression to chronic angle closure glaucoma. Widening of the anterior chamber angle may also be responsible for the decrease in intraocular pressure that is often observed 

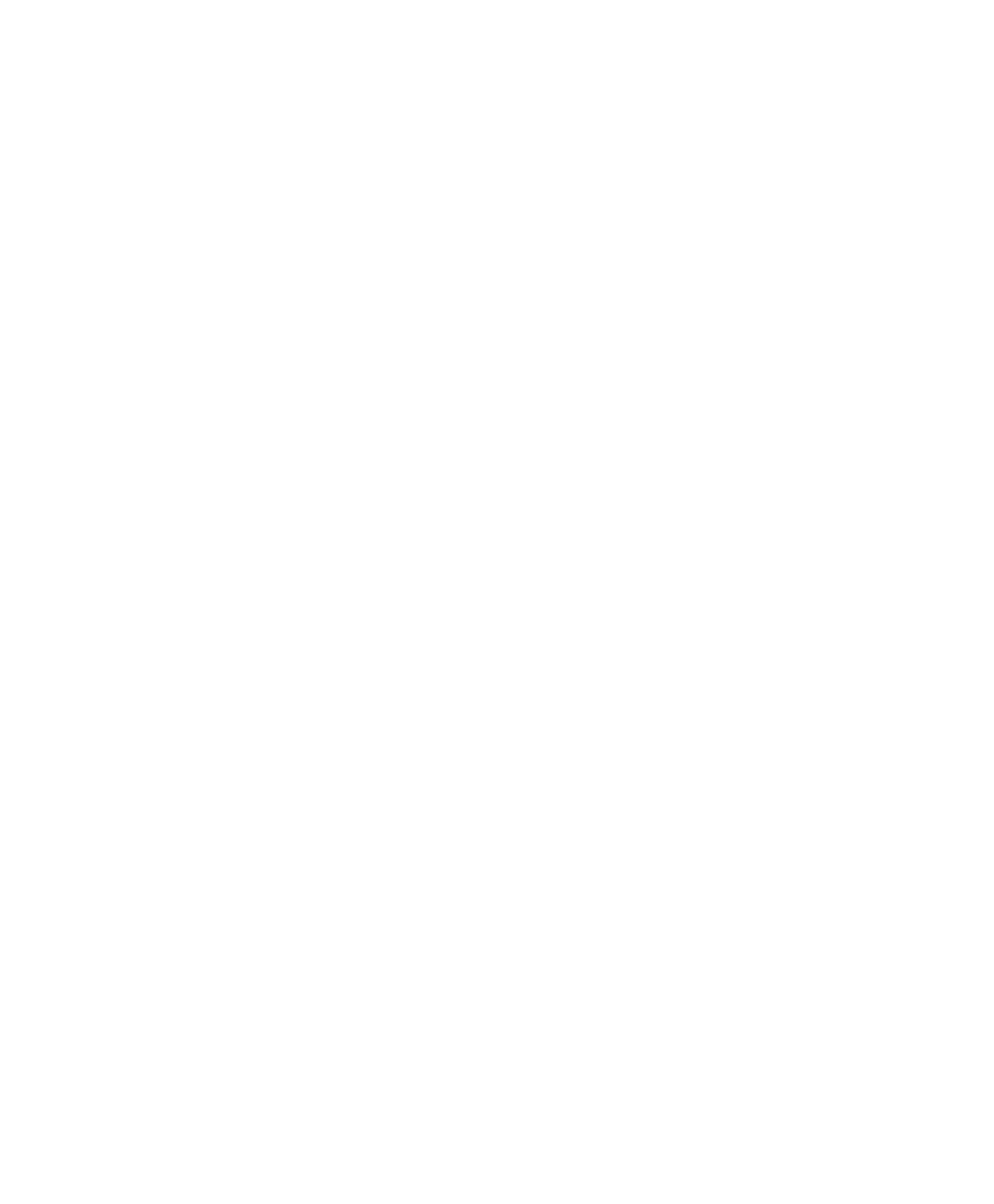

Figs 10A to C: Anterior segment optical coherence tomography showing the anterior chamber configuration of a patient with primary angle closure $(A)$ who first underwent laser peripheral iridotomy (B), followed by phacoemulsification with intraocular lens implant (C). While his anterior chamber angles were not sufficiently widened with laser iridotomy, phacoemulsification was successful in widening the angles and deepening the anterior chamber

in the postoperative period after phacoemulsification. In established angle closure glaucoma however, and where factors other than pupil block, angle crowding and lens are responsible for angle closure, lens extraction alone may not be adequate, and combined filtration surgery should be considered.
Phacoemulsification in angle closure glaucoma poses many challenges to the surgeon. However, with careful preoperative evaluation, meticulous surgical planning, a gentle surgical technique and vigilant postoperative monitoring, surgery can be highly successful in these patients resulting in good visual acuity and intraocular pressure control. 


\section{REFERENCES}

1. Teekhasaenee C, Ritch R. Combined phacoemulsification and goniosynechialysis for uncontrolled chronic angle closure glaucoma after acute angle closure glaucoma. Ophthalmology 1999;106:669.

2. Lai JS, Tham CC, Chua JK, et al. Efficacy and safety of inferior 180 degrees goniosynechialysis followed by diode laser peripheral iridoplasty in the treatment of chronic angle closure glaucoma. J Glaucoma 2000;9:388.

3. Simmons RJ. Malignant glaucoma. Br J Ophthalmol 1972;56:263.

4. Lowe RF. Malignant glaucoma related to primary angle closure glaucoma. Aust J Ophthalmol 1979;7:11.

5. Hayashi K, Hayashi H, Nakao F, et al. Changes in anterior chamber angle width and depth after intraocular lens implantation in eyes with glaucoma. Ophthalmology 2000;107:698-703.

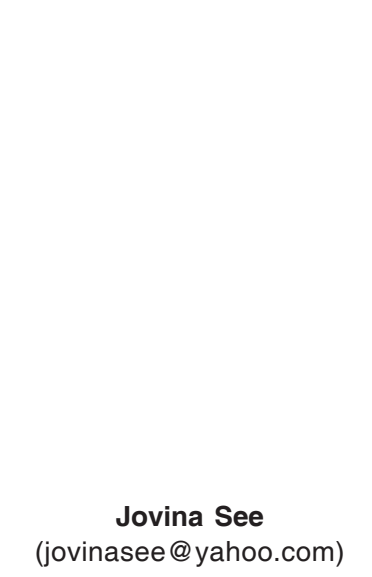

"It is pleasing to human vanity to believe that one suffers because of ones virtue; but not until a man has extirpated every sickly, bitter and impure thought from his mind, and washed every sinful stain from his soul, can he be in apposition to know and declare that his sufferings are the result of his good, and not of his bad qualities; and on the way to, yet long before he has reached, that supreme perfection, he will have found, working in his mind and life, the great law which is absolutely just, and which cannot, therefore give good for evil, evil for good. Possessed of such knowledge, he will then know, looking back upon his past ignorance and blindness, that his life is, and always was, just ordered, and that his past experiences, good and bad, were the equitable outworking for his evolving,yet unevolved self.” 